\title{
Zur Kenntnis der Magnesiumcarbide
}

\section{Doctoral Thesis}

Author(s):

Irmann, Frank

Publication date:

1949

Permanent link:

https://doi.org/10.3929/ethz-a-000097553

Rights / license:

In Copyright - Non-Commercial Use Permitted 


\title{
ZUR KENNTNIS \\ DER MAGNESIUMCARBIDE
}

\author{
Von der \\ EIDGENÖSSISCHEN TECHNISCHEN \\ HOCHSCHULE IN ZURICH
}

\author{
zur Erlangung \\ der Würde eines Doktors \\ der technischen Wissenschaften \\ genehmigte \\ PROMOTIONSARBEIT \\ vorgelegt von \\ FRANK IRMANN \\ von Neuhausen am Rheinfall
}

Referent: Herr Prof. Dr. W. D. Treadwell

Korreferent: Herr Prof. Dr. G. Trümpler

Grütli-Buchdruckerei Zürich 1949 


\section{ZUSAMMENFASSUNG}

1. Die Bildung der Magnesiumcarbide aus dem Metall und verschiedenen Kohlenwasserstoffen, sowie aus Magnesiumdiäthyl und Aethin wurde untersucht und die Temperaturbedingungen ermittelt, was zu einer Bestätigung und Ergänzung der Literaturangaben führte.

2. Um die bei der Hydrolyse der beiden Carbide entstehenden Gase, Aethin $\left(\mathrm{C}_{2} \mathrm{H}_{2}\right)$ und Propin $\left(\mathrm{C}_{3} \mathrm{H}_{4}\right)$, zu bestimmen, wurde eine Adsorptionsmethode ausgearbeitet.

3. Es wurden Präparate mit $70 \% \quad \mathrm{MgC}_{2}$ bzw. $85 \%$ $\mathrm{Mg}_{2} \mathrm{C}_{3}$ hergestellt.

4. Die Lösungswärmen beider Carbide in Salzsäure wurden gemessen. Daraus ergaben sich die Bildungswärmen aus den Elementen als endotherm, nämlich zu:

$$
\begin{array}{ll}
\mathrm{MgC}_{2}: \Delta \mathrm{H}_{298}=+21 \pm 5 \mathrm{kcal} / \mathrm{g} \text {-A tom } \mathrm{Mg} \\
\mathrm{Mg}_{2} \mathrm{C}_{3}: \Delta \mathrm{H}_{298}=+9 \pm 4 \mathrm{kcal} / \mathrm{g} \text {-Atom Mg. }
\end{array}
$$

5. Debye-Scherrer-Diagramme von $\mathrm{MgC}_{2}$ und $\mathrm{Mg}_{2} \mathrm{C}_{3}$ konnten tetragonal bzw. hexagonal indiziert werden. Ueber die Struktur des $\mathrm{MgC}_{2}$ ließ sich eine Vermutung aussprechen.

Vorliegende Arbeit konnte mit Unterstützung des Arbeitsbeschaffungskredites der ETH. durchgeführt werden, wofür ich hier meinen Dank aussprechen möchte. 